\title{
Exome sequencing reveals a de novo PRKG1 mutation in a sporadic patient with aortic dissection
}

\author{
Wenwen Zhang ${ }^{1,2+}$, Qian $\mathrm{Han}^{3 \dagger}$, Zhao $\mathrm{Liu}^{3}$, Wei Zhou', Qing Cao ${ }^{2}$ and Weimin Zhou ${ }^{{ }^{*}}$ (D)
}

\begin{abstract}
Background: Thoracic aortic aneurysm and dissection (TAAD) is a common condition associated with high mortality. It is predominantly inherited in an autosomal dominant manner with reduced penetrance and variable expression. The genetic basis of the majority of TAAD cases remains unknown.

Case presentation: We described a 53 years old male presented with abdominal aortic dissection as well as aortic tortuosity. To investigate the genetic basis of the clinical presentation, whole-exome sequencing was performed. Exome sequencing identified a de novo heterozygous undescribed mutation in the PRKG1 gene (NM_001098512.2: c.1108 G > A), predicted to cause the missense change p.Gly370Ser in the ATP binding motif of the protein. This mutation was not reported in the dbSNP, 1000 Genome Project, and Exome sequencing databases. Furthermore, the Glycine370 residue of PRKG1 is highly conserved among various species and it is predicted to be damaging by multiple in silico programs, suggesting that this substitution may cause a major disruption of protein function. To our knowledge, this is the second reported mutation locus of PRKG1 accounting for the disease.

Conclusions: Our study expands the mutation spectrum of PRKG1 and clinical phenotype of mutation-carriers. Screening for PRKG1 mutations should be considered in patients with unexplained aortic disease, and identification of the causative gene will aid in individualized, gene-tailored management.
\end{abstract}

Keywords: Aortic dissection, PRKG1 gene, Whole-exome sequencing, Binding motif

\section{Background}

Thoracic aortic aneurysm and dissection (TAAD) remains a significant clinical challenge due to its high morbidity and mortality [1]. Previous studies have demonstrated that approximately $20 \%$ of TAAD patients have a family history, indicating a genetic component to the disease [2]. It is predominantly inherited in an autosomal dominant fashion with reduced penetrance and variable expression, showing significant genetic $(>20$ genes) and clinical (location, severity and age of onset) heterogeneity [3]. TAAD patients caused by different genes have distinct clinical courses and risk of rupture or dissection, influencing intervening measure and timing [4]. The identification of specific mutated genes in

\footnotetext{
* Correspondence: docncu@163.com

'Wenwen Zhang and Qian Han contributed equally to this work.

'Department of Vascular Surgery, The Second Affiliated Hospital of

Nanchang University, No 1\#, Minde Road, Nanchang, China

Full list of author information is available at the end of the article
}

patients with TAAD is therefore crucial because it permits individualized, gene-tailored therapy.

The majority of genes associated with the development of TAAD encode proteins involved in the extracellular matrix maintenance, smooth muscle cell (SMC) contraction or metabolism, or canonical transforming growth factor- $\beta$ signaling pathway [5]. Dysfunction of SMC contractile apparatus impairs resilience of aorta in the context of pulsatile blood flow and shear stress. Accordingly, mutations in genes encoding smooth muscle-specific isoform of $\alpha$-actin (ACTA2) and myosin heavy chain dimer (MYH11) are responsible for roughly a quarter of familial TAAD patients [6, 7]. Mutations in MYLK are another rare cause of familial TAAD [8]. MYLK encodes smooth muscle myosin light chain kinase, which drives SMC contraction through phosphorylation of the regulatory light chain on the thick filaments.

In 2013, a single heterozygous mutation in the gene encoding cGMP-dependent protein kinase 1 (PRKG1; 
protein PKG-1; chromosomal 10q11.2-q21.1) was demonstrated to cause TAAD [9]. PKG-1 is activated on binding of cGMP and plays an important role in SMC relaxation. The causal mutation, pArg177Gln, is located in the high-affinity cGMP binding site, and such alteration in this domain abolished binding of cGMP. Paradoxically, the mutation resulted in the enzyme being constitutively active even in the absence of cGMP, suggesting the mechanism of gain-of-function. Individuals harboring this mutation have aortic dissections at a relatively young age (15-51 years) at diameters ranging 4.3 to $5.7 \mathrm{~cm}$. Aortic tortuosity and hypertension are also noted in some mutation-carriers.

Here, we identified an individual with abdominal aortic dissection characterized by tortuosity, as well as congenital visual impairment caused by keratoconus. Whole-exome sequencing revealed a heterozygous sequence variation in PRKG1 (c.1108 G > A, p.Gly370Ser). The mutation lies in a conserved motif with pivotal function, and multiple lines of evidence support its pathogenic role. To our knowledge, this is the second reported mutation locus of PRKG1 accounting for the disease.

\section{Case presentation}

The index patient was a male of 53 years who was referred to our department complaining of abdominal pain, which failed to subside after administration of antispasmodic agents. Computed Tomography Angiography
(CTA) showed abdominal aortic dissection originating distal to the renal artery and extending to the iliac arteries, with the celiac artery and the superior mesenteric artery involved (Fig. 1a). Tortuosity of aorta was also observed (Tortuosity was defined as the presence of at least two bends $>70^{\circ}$ proximal to the target lesion or one proximal bend $>90^{\circ}$ ) (Fig. 1b). His medical history was significant for hypertension for 4 years treated with $\mathrm{Ca}$ antagonists. He had ischemic heart disease, including history of acute myocardial infarction at age 51 with no stents implanted. His past medical history also included moderate scoliosis diagnosed at age 10 .

On physical examination, clinical features reminiscent of connective tissue disorders were not remarkable, except for congenital visual impairment. Subsequent corneal topography measurements were taken and keratoconus was diagnosed. Family history of aorta diseases was negative, as his parents displayed normal aorta on contrast-enhanced CT. The family provided written informed consent for clinical and molecular analyses, and the research protocol was approved by the Institute Review Board of the Second Affiliated Hospital of Nanchang University.

The patient was first diagnosed as arterial tortuosity syndrome (ATS), due to strikingly overlapped clinical features (aorta dissection, tortuosity and keratoconus) [10]. The coding exons and intronic boundaries of the $S L C 2 A 10$ gene was first screened by direct Sanger sequencing, with no causative mutation found in the gene.
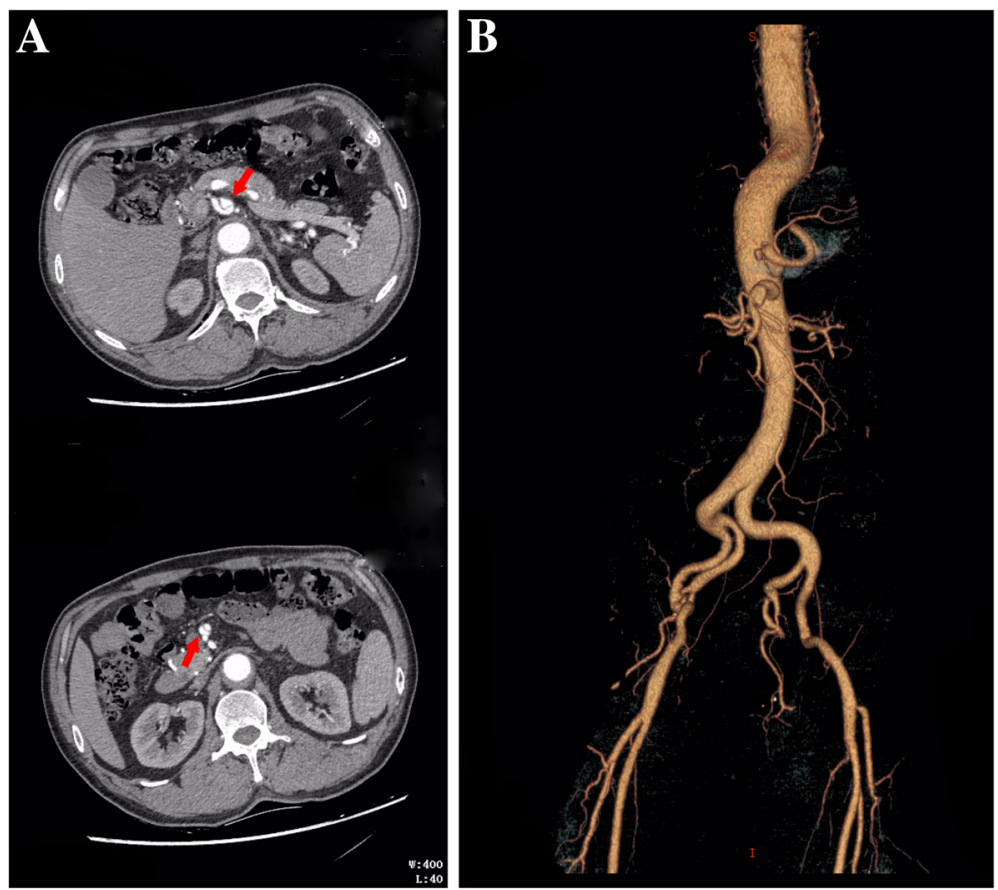

Fig. 1 Radiographic findings of the patient. a Multi-slice computed tomography shows abdominal aortic dissection with the celiac artery and the superior mesenteric artery (Red arrow) involved. b 3D-reconstructed computed tomography angiogram shows tortuosity of abdominal and iliac aorta 
To investigate the genetic basis of the disease, whole-exome sequencing was performed as previously described [11]. Genomic DNA was captured using the Agilent SureSelect V6 enrichment kit and sequenced on an Illumina HiSeq 2500 as 150-bp paired-end runs. In accordance with the Genome Analysis ToolKit's (GATK) best practices guidelines, we mapped sequence reads to the human reference genome (hg19) using the Burrows-Wheeler Aligner (v.0.7.8), removed duplicate reads (Picard v.1.111), and identified SNPs and Indels (SAMtools v.1.0). ANNOVAR (v. 2015Dec14) was utilized to annotate the detected variations.

On average, $99.8 \%$ of short reads were mapped, reaching an average $\sim 130$-fold depth of coverage. $99.6 \%$ of targeted regions were covered by at least 10 unique reads. A summary of the filtering strategy of this study is presented in Table 1. Exome data yielded 47 likely pathogenic variants after sequential filtering process. Among them, we focused on variants of known causal genes or genes implicated in aorta diseases. Finally, an unreported heterozygous c.1108 G > A (p.Gly370Ser) missense mutation in the 10th coding exon of the PRKG1 gene (NM_001098512.2) was identified.

Bidirectional Sanger sequencing confirmed the mutation in the patient. By employing short tandem repeat multiplex assay, we confirmed the biological association of the father and mother with the patient. Its absence in both parents validated the de novo status of the mutation (Fig. 2a). The c.1108 G > A variant was absent from dbSNP144, the 1000G, ESP, ExAC and our in-house variant databases. Furthermore, this variant was predicted as damaging according to online prediction tools such as SIFT, Polyphen 2 and Mutation Taster. Moreover, the CADD C-score of the mutation was 33, strongly predicting that the p.Gly370Ser as deleterious (scores $>15$ are deleterious).

The main gene product of PRKG1 expressed in the aorta, PKG-1 $\alpha$, is a homodimer with the N-terminal regulatory and $\mathrm{C}$-terminal catalytic domains (Fig. 2b) [12]. Located in the ATP binding region, the glycine-rich GXGGXXGXV motif is capable to bind the adenine ring of ATP by forming a hydrophobic pocket [13]. The variant identified in this study is located in the third highly

Table 1 Filtering strategy of the patient

\begin{tabular}{ll}
\hline Filtering criteria & $\begin{array}{l}\text { Number of } \\
\text { variants }\end{array}$ \\
\hline Total number of variants & 169,956 \\
Missense, nonsense, splice-site or frameshifit & 10,363 \\
variants & \\
MAF $<1 \%$ in the 1000G, ESP, ExAC & 520 \\
Absence in our in-house databases & 245 \\
Prediction in silico (SIFT, Polyphen2, MutationTaste) & 61 \\
CADD score $>15$ & 47 \\
\hline
\end{tabular}

conserved glycine residue. In addition, the replacement of hydrophobic glycine residue by hydrophilic serine may cause the conformational change of the pocket, disrupting its binding capability and thereby impairing protein function. According to the latest American College of Medical Genetics and Genomics (ACMG) guidelines for the interpretation of sequence results, this variant in PRKG1 gene fulfills PM1, PM2, PP3 and PS2 and is therefore regarded as likely pathogenic [14].

\section{Discussion and conclusions}

TAAD is a heterogenous monogenic disorder characterized by overlapping manifestations and variable expression. It is therefore challenging to identify the underlying gene defect just based on its clinical features. Conventional Sanger sequencing is time-consuming and laborious if multiple disease-causing genes exist. In this study, by exome sequencing we successfully identified the culprit mutation of in a patient who first had a diagnosis of ATS, given the particularly fitting clinical features. Molecular analysis revealed a heterozygous de novo missense mutation c. $1108 \mathrm{G}>\mathrm{A}$ (p.Gly370Ser) in the PRKG1 gene.

Multiple lines of evidence support this variant as a pathogenic variant causing the patient's clinical presentation. First, no missense variants have been reported in the public variation databases at this position. Second, the p.Gly370Ser is located within the glycine-rich ATP binding region, a highly-conserved motif across eukaryotic protein kinases. The motif functions as a hydrophobic pocket capable of binding the adenine ring of ATP, and substitution of glycine for hydrophilic serine at this position is unlikely to be tolerated. Third, in silico analysis using multiple online tools shows consistently that the p.Gly370Ser substitution is predicted to be damaging to protein function. Amino acid property probably plays a key role in the correct folding of this region into the hydrophobic pocket. It is notable that not only is residue 370 the hydrophobic glycine, but that the surrounding residues are also highly conserved non-polar residues [13]. The substitution of Gly370 by the polar serine is likely to cause local structure instability. Taken together, it is very likely that the p.Gly370Ser substitution will cause disruption of the hydrophobic pocket, and, since this has been shown to be indispensable for interaction with ATP, perturbation of protein function.

Guo et al. identified a recurrent mutation, p.Arg177Gln, in four unrelated family of TAAD [9]. The mutation segregated with the disease in each family and was not present in controls. The p.Arg177Gln is located in the high-affinity cGMP binding site, another conserved domain with pivotal function. In vitro function studies demonstrated that it resulted in constitutive kinase activity even in the absence of cGMP, indicating 


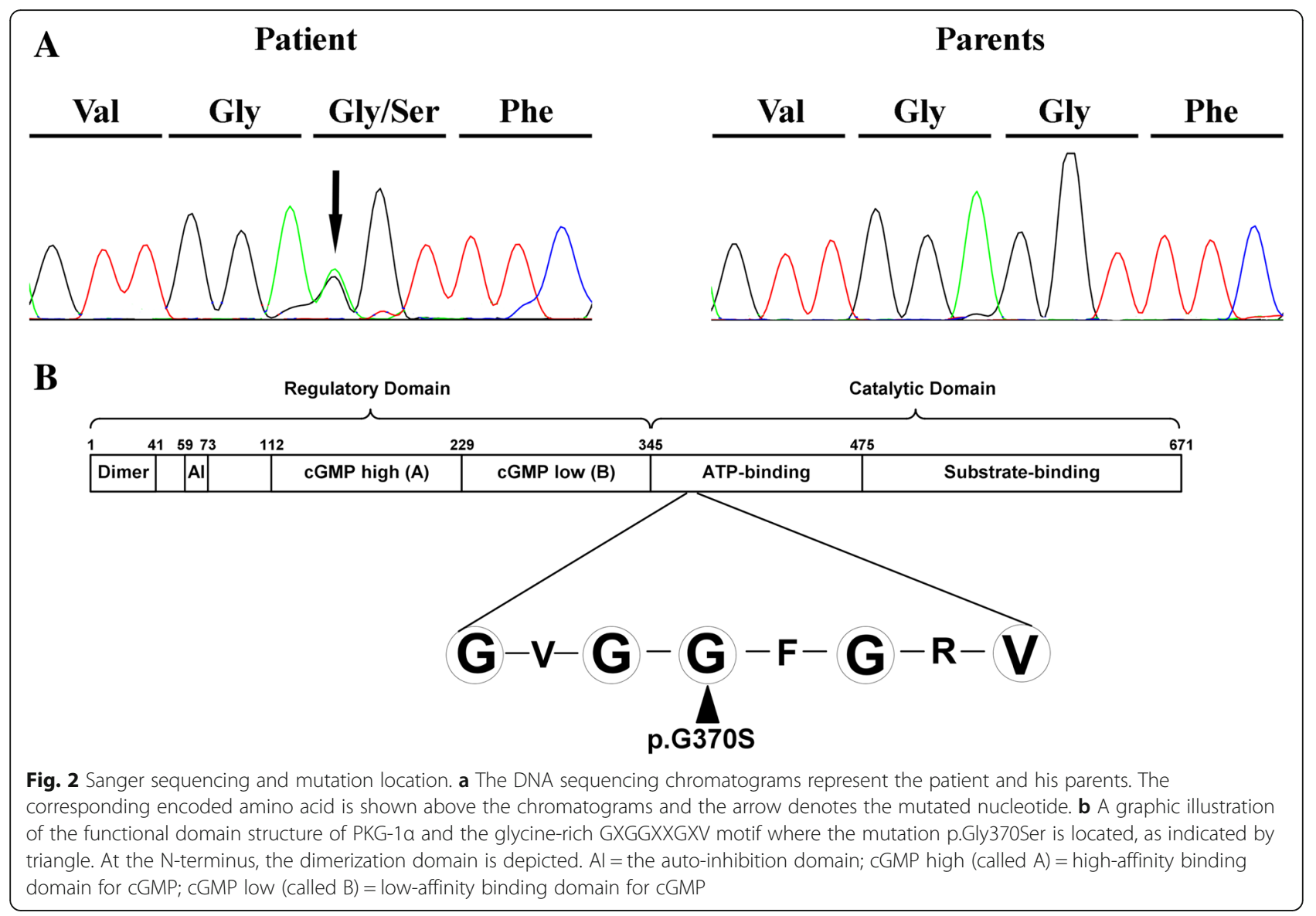

gain-of-function for the mutation. Mutation-carriers presented with either thoracic aortic dissection or aortic root dilatation, with thoracic aortic disease fully penetrate in those aged over 18 years old. Some patients also suffered from abdominal aortic dissection and showed aorta tortuosity, as reported in our case. Gago-Diaz et al. investigated the molecular defect in a large TAAD pedigree and found the same mutation, suggesting that the p.Arg177Gln may be a mutation hotspot [15]. The penetrance of TAAD in this family was nearly complete, except for a 33-year-old female. Apart from aortic features, other extra-aortic connective tissue manifestations were also found in a subset of patients, which includes scoliosis, wrist and thumb signs, skin striae, myopia, pectus carinatum/excavatum deformity, chest asymmetry. But none of them fulfilled diagnostic criteria of the main connective tissue syndromes.

Ocular problem constitutes an important feature in a considerable proportion of patients harboring mutations in TAAD disease-causing genes [16]. For example, ectopia lentis and myopia were frequently observed in $F B N 1$ mutation-carriers; myopia in SKI; mydriasis in ACTA2 [17]. The revised Ghent nosology puts more weight on ectopia lentis in the diagnosis of Marfan syndrome caused by FBN1 mutation [18]. The occurrence of ocular problem in TAAD patients can to some degree aid in the recognition of the underlying genes. A special form of ocular disorders, keratoconus, is very rare in TAAD patients. ATS is an uncommon autosomal recessive disorder characterized by widespread arterial involvement with tortuosity, elongation, and aneurysms of the large and middle-sized arteries, with some patients exhibiting keratoconus phenotype [10]. The inheritance model and clinical features of our case made us suspect the diagnosis of ATS, while genetic testing of SLC2A10 yielded negative results. Our study is the first to show the phenotype of keratoconus in PRKG1 mutation-carriers. However, whether it is associated with PRKG1 mutation or just accidental finding requires further research.

Based on the anatomic location, aortopathy is subdivide into abdominal and thoracic aortic lesions. Abdominal aortic lesions are more common and tend to occur at later age. They seem to be multifactorial and associated with common cardiovascular risk factors. So far, no disease-causing variants have been identified to account for abdominal aortic diseases. However, thoracic aortic lesions have a greater heritability and often show family clustering. Indeed, a substantial proportion of them are manifestations of part of Mendelian connective tissue disorder. Apart from thoracic involvement, some 
patients also exhibit non-thoracic lesions, such as abdominal and intracranial diseases. Their features include early-onset and a lack of common cardiovascular risk factors. Their underlying genetic architecture often warrants further investigation.

In conclusion, we report a novel case affected with aortic dissection and have identified a novel likely pathogenic variant in the ATP binding domain of PRKG1, which provides further evidence for the role of this gene in causing TAAD. Adding to previous reports, our study underlines the need to include this gene among the candidate genes tested in patients affected with unexplained aortic disease. Identification of the causative gene allows identification of additional risky family members and gene-based management of the carriers.

\section{Abbreviations}

ACMG: American college of medical genetics and genomics; ATS: Arterial tortuosity syndrome; CTA: Computed tomography angiography; GATK: Genome analysis toolkit; SMC: Smooth muscle cell; TAAD: Thoracic aortic aneurysm and dissection

\section{Acknowledgements}

We would like to thank the family for their participation in the study.

\section{Funding}

This study was supported by grants from the National Natural Science Foundation of China (81660086).

\section{Availability of data and materials}

The data and materials that support the findings of this study are available on request from the corresponding author [WMZ]. The data are not publicly available due to them containing information that could compromise research participant privacy/consent.

\section{Authors' contributions}

Recruited and phenotyped the participants: WWZ, WMZ. Performed molecular genetic experiments: QH, ZL, WZ. Analyzed the data: WWZ, QH, QC. Wrote the manuscript: WWZ, WMZ. All authors read and approved the final manuscript.

\section{Ethics approval and consent to participate}

Ethical approval was obtained from the institutional review board, The Second Affiliated Hospital of Nanchang University. After explanation of the possible consequences of the study, written informed consent was obtained from all study participants.

\section{Consent for publication}

Consent for publication of the case was obtained for each participant, including case description, clinical data and images.

\section{Competing interests}

The authors declare that they have no competing interest.

\section{Publisher's Note}

Springer Nature remains neutral with regard to jurisdictional claims in published maps and institutional affiliations.

\section{Author details}

'Department of Vascular Surgery, The Second Affiliated Hospital of Nanchang University, No 1\#, Minde Road, Nanchang, China. ${ }^{2}$ Key Laboratory of Molecular Medicine of Jiangxi Province, Nanchang, Jiangxi, China. ${ }^{3}$ Department of Vascular Surgery, Nanjing Drum Tower Hospital, the Affiliated Hospital of Nanjing University Medical School, Nanjing, Jiangsu, China.
Received: 17 April 2018 Accepted: 13 December 2018 Published online: 22 December 2018

\section{References}

1. Criado FJ. Aortic dissection: a 250-year perspective. Tex Heart Inst J. 2011;38: 694-700.

2. Biddinger $A$, Rocklin M, Coselli J, Milewicz DM. Familial thoracic aortic dilatations and dissections: a case control study. J Vasc Surg. 1997;25:506-11.

3. Albornoz G, Coady MA, Roberts M, Davies RR, Tranquilli M, Rizzo JA, Elefteriades JA. Familial thoracic aortic aneurysms and dissections--incidence, modes of inheritance, and phenotypic patterns. Ann Thorac Surg. 2006;82:1400-5.

4. Milewicz D, Hostetler E, Wallace S, Mellor-Crummey L, Gong L, Pannu H, Guo DC, Regalado E. Precision medical and surgical management for thoracic aortic aneurysms and acute aortic dissections based on the causative mutant gene. J Cardiovasc Surg. 2016;57:172-7.

5. Verstraeten A, Luyckx I, Loeys B. Aetiology and management of hereditary aortopathy. Nat Rev Cardiol. 2017;14:197-208.

6. Zhu L, Vranckx R, Khau Van Kien P, Lalande A, Boisset N, Mathieu F, Wegman M, Glancy L, Gasc JM, Brunotte F, Bruneval P, Wolf JE, Michel JB, Jeunemaitre $X$. Mutations in myosin heavy chain 11 cause a syndrome associating thoracic aortic aneurysm/aortic dissection and patent ductus arteriosus. Nat Genet. 2006:38:343-9.

7. Guo DC, Pannu H, Tran-Fadulu V, Papke CL, Yu RK, Avidan N, Bourgeois S, Estrera AL, Safi HJ, Sparks E, Amor D, Ades L, McConnell V, Willoughby CE, Abuelo D, Willing M, Lewis RA, Kim DH, Scherer S, Tung PP, Ahn C, Buja LM, Raman CS, Shete SS, Milewicz DM. Mutations in smooth muscle alpha-actin (ACTA2) lead to thoracic aortic aneurysms and dissections. Nat Genet. 2007; 39:1488-93.

8. Wang L, Guo DC, Cao J, Gong L, Kamm KE, Regalado E, Li L, Shete S, He WQ, Zhu MS, Offermanns S, Gilchrist D, Elefteriades J, Stull JT, Milewicz DM. Mutations in myosin light chain kinase cause familial aortic dissections. Am J Hum Genet. 2010;87:701-7.

9. Guo DC, Regalado E, Casteel DE, Santos-Cortez RL, Gong L, Kim JJ, Dyack S, Horne SG, Chang G, Jondeau G, Boileau C, Coselli JS, Li Z, Leal SM, Shendure J, Rieder MJ, Bamshad MJ, Nickerson DA, Gen TACRC, National Heart L, Blood Institute Grand Opportunity Exome Sequencing P, Kim C, Milewicz DM. Recurrent gain-of-function mutation in PRKG1 causes thoracic aortic aneurysms and acute aortic dissections. Am J Hum Genet. 2013;93:398-404.

10. Coucke PJ, Willaert A, Wessels MW, Callewaert B, Zoppi N, De Backer J, Fox JE, Mancini GM, Kambouris M, Gardella R, Facchetti F, Willems PJ, Forsyth R, Dietz HC, Barlati S, Colombi M, Loeys B, De Paepe A. Mutations in the facilitative glucose transporter GLUT10 alter angiogenesis and cause arterial tortuosity syndrome. Nat Genet. 2006:38:452-7.

11. Zhang W, Zeng Q, Xu Y, Ying H, Zhou W, Cao Q, Zhou W. Exome sequencing identified a novel SMAD2 mutation in a Chinese family with early onset aortic aneurysms. Clin Chimica Acta. 2017:468:211-4.

12. Tamura N, Itoh H, Ogawa $Y$, Nakagawa O, Harada M, Chun TH, Suga S, Yoshimasa T, Nakao K. CDNA cloning and gene expression of human type lalpha CGMP-dependent protein kinase. Hypertension. 1996;27:552-7.

13. Alverdi V, Mazon H, Versluis C, Hemrika W, Esposito G, van den Heuvel R, Scholten A, Heck AJ. cGMP-binding prepares PKG for substrate binding by disclosing the C-terminal domain. J Mol Biol. 2008;375:1380-93.

14. Richards S, Aziz N, Bale S, Bick D, Das S, Gastier-Foster J, Grody WW, Hegde M, Lyon E, Spector E, Voelkerding K, Rehm HL, Committee ALQA. Standards and guidelines for the interpretation of sequence variants: a joint consensus recommendation of the American College of Medical Genetics and Genomics and the Association for Molecular Pathology. Genet Med. 2015;17:405-24.

15. Gago-Diaz M, Blanco-Verea A, Teixido G, Huguet F, Gut M, Laurie S, Gut I, Carracedo A, Evangelista A, Brion M. PRKG1 and genetic diagnosis of earlyonset thoracic aortic disease. Eur J Clin Investig. 2016;46:787-94.

16. Bradley TJ, Bowdin SC, Morel CF, Pyeritz RE. The expanding clinical Spectrum of Extracardiovascular and cardiovascular manifestations of heritable thoracic aortic aneurysm and dissection. Canad J Cardiol. 2016;32:86-99.

17. Milewicz DM, Ostergaard JR, Ala-Kokko LM, Khan N, Grange DK, MendozaLondono R, Bradley TJ, Olney AH, Ades L, Maher JF, Guo D, Buja LM, Kim D, Hyland JC, Regalado ES. De novo ACTA2 mutation causes a novel syndrome of multisystemic smooth muscle dysfunction. Am J Med Genet A. 2010;152A:2437-43.

18. Loeys BL, Dietz HC, Braverman AC, Callewaert BL, De Backer J, Devereux RB, Hilhorst-Hofstee Y, Jondeau G, Faivre L, Milewicz DM, Pyeritz RE, Sponseller PD, Wordsworth P, De Paepe AM. The revised Ghent nosology for the Marfan syndrome. J Med Genet. 2010;47:476-85. 